\title{
SOFTWARE ARCHITECTURE DESIGN OF GIS WEB SERVICE AGGREGATION BASED ON SERVICE GROUP
}

\author{
LIU Jian-chuan*, YANG Jun, TAN Ming-jian, GAN Quan \\ Sichuan Geomatics Center, Chengdu 610041, China
}

Keywords: GIS; Web; Design; Software; Architecture; Geography

\begin{abstract}
:
Based on the analysis of research status of domestic and international GIS web service aggregation and development tendency of public platform of GIS web service, the paper designed software architecture of GIS web service aggregation based on GIS web service group. Firstly, using heterogeneous GIS services model, the software architecture converted a variety of heterogeneous services to a unified interface of GIS services, and divided different types of GIS services into different service groups referring to description of GIS services. Secondly, a service aggregation process model was designed. This model completed the task of specific service aggregation instance, by automatically selecting member GIS Web services in the same service group. Dynamic capabilities and automatic adaptation of GIS Web services aggregation process were achieved. Thirdly, this paper designed a service evaluation model of GIS web service aggregation based on service group from three aspects, i.e. GIS Web Service itself, networking conditions and service consumer. This model implemented effective quality evaluation and performance monitoring of GIS web service aggregation. It could be used to guide the execution, monitor and service selection of aggregation process. Therefore, robustness of aggregated GIS web service was improved. Finally, the software architecture has been widely used in public platform of GIS web service and a number of geo-spatial framework constructions for digital city in Sichuan Province, and aggregated various GIS web services such as World Map(National Public Platform of Geo-spatial Service), ArcGIS, SuperMap, MapGIS, NewMap etc. Applications of items showed that this software architecture was practicability.
\end{abstract}

\section{INTRODUCTION}

In China, national, provincial and municipal public platform of geo-spatial services are orderly building. It is the focus of public service platform research that the national, provincial and municipal distributed GIS web services aggregate together and achieve vertical interoperability. Through investigation, National Public Platform of Geo-spatial Service (World Map) used software of GeoGlobe as the Based GIS software. However, because their basis and conditions of construction are different, provincial and municipal public service platforms used and will use a variety of basic GIS software, such as ArcGIS, SuperMap, MapGIS, NewMap etc. Data, service interface and service management of these software have their own characteristics. Being different from ordinary, these heterogeneous GIS web services deal with complex distributed spatial processing functions and distributed spatial data. Therefore, they have compute-intensive and data-intensive features. Any service exceptions and errors of member services in GIS web service aggregation could cause the failure of the implementation of aggregation processes and result in great waste computing resources.

Based on this, firstly, classifying a variety of the same GIS web services as a group of GIS web service, the paper designed a software architecture of GIS web service aggregation based on GIS web service group. Using interfaces of web service, this software architecture separated service aggregation process model from service distribution and management software. Users could not need to understand what the underlying member services have been called, only a URL would be able to call GIS web service aggregation. Secondly, a service evaluation model of GIS web service aggregation was designed in detail. It described and recorded QoS (Quality of Service) of member services from three aspects, i.e. GIS Web Service itself, networking conditions and service consumer. Using this model, aggregation service engine chose the best member service.
Therefore, robustness of aggregated GIS web service was improved.

\section{GIS SERVICE AGGREGATION SOFTWARE ARCHITECTURE DESIGN}

\subsection{GIS Service Group Description}

To aggregate different GIS web services, the most important task is to divide different types of GIS services into different service groups according to the function and scope of services. The so-called service group (SG), is a set of GIS web services, which consist of different service providers, have the same interface and achieve the same functionality and the same range of services.

Service group can be expressed as a seven-tuple. $\mathrm{SG}=(\mathrm{ID}$, Name, Description, Operation, Input, Output, Capability).

\section{$>$ ID: identifier of SG}

$>$ Name: name of SG

$>$ Description: description of SG

$>$ Operation: operation names of SG

Input: Input parameters of service in SG

$>$ Output: output parameters of service in SG

$>$ Capability: capability description of service in SG (Geo-spatial data services, for example, included extent of data, type of data, fields of data etc.)

The services in the same service group have the same function and interface. The difference is the web sites and QoS properties (non-functional properties) of the various services. Every GIS web service in SG is called member service (MS). A general description of MS can be expressed as an eight-tuple. MS= (ID, Name, SGID, Description, Url, Token, Range, Qos).

\section{$>$ ID: identifier of MS}


Name: name of MS

$>$ Description: description of MS

$>$ SGID: SG identifier of MS

$>$ Url: url of MS

Token: authorized identifier of MS

$>$ Range: service range of MS

$>$ QoS: description of MS, and will been expatiated in 3.1 .

\subsection{Service Aggregation Software Architecture Design}

Based on description of MS and SG, service aggregation software architecture was designed (shown in Fig.1.). It was divided into several parts: Heterogeneous service transformation model, Abstract service interface, Service registration center, GIS Service group, Service aggregation process model.

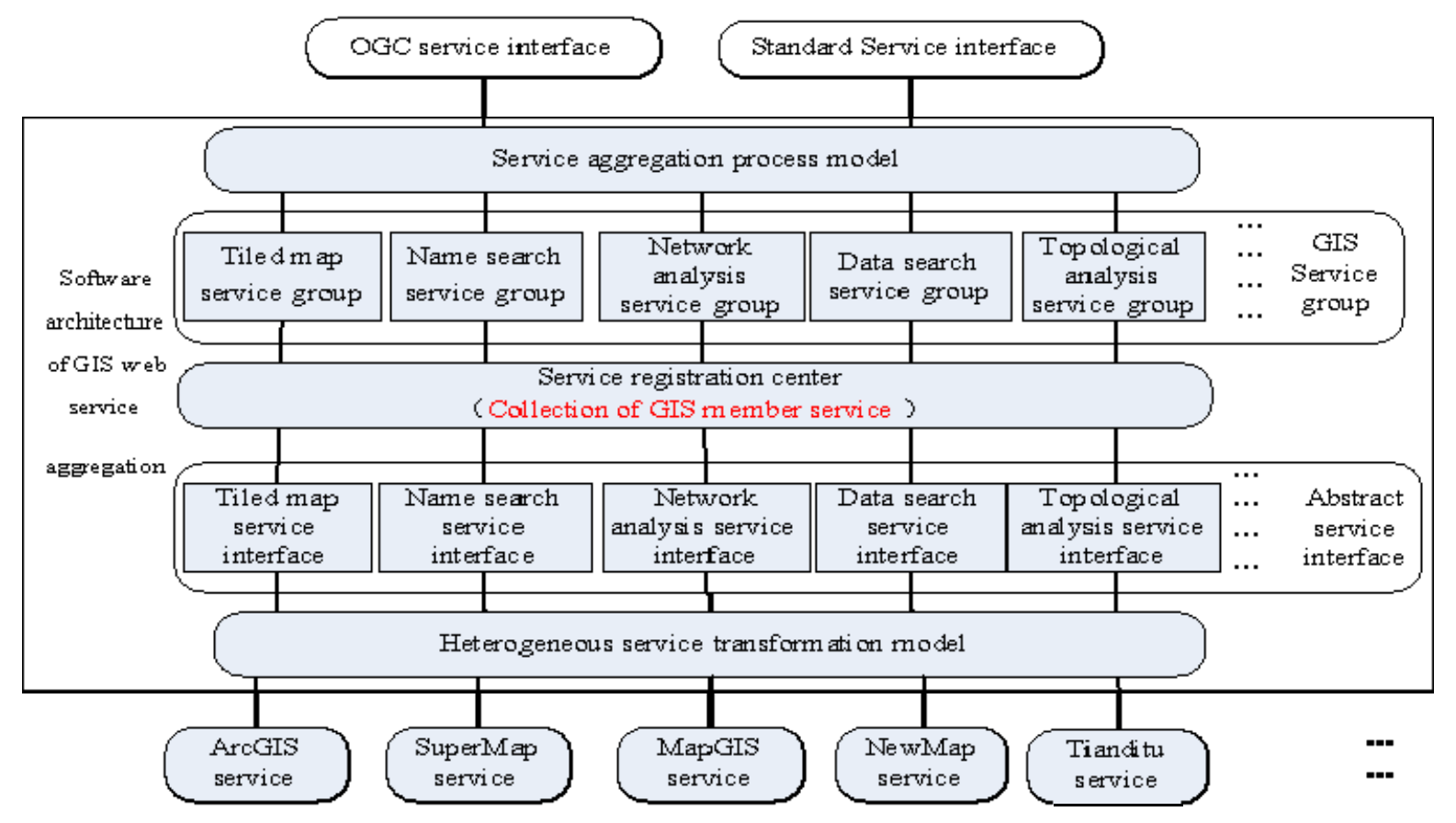

Fig.1. Software architecture diagram of GIS service aggregation based on SG

Heterogeneous service transformation model: It is often encountered problem that different software companies (such as ArcGIS, SuperMap ,MapGIS, NewMap, World Map etc.) defined different service interfaces of the same type of service. If these services are aggregated directly, difficulty and workload of programming are Considerable. Therefore, heterogeneous service transformation model has been designed. Using certain algorithms and dynamic library, it converted heterogeneous service interface to abstract GIS service interface, and greatly simplified the mapping process of services. The difficulty of service aggregation has been reduced.

Abstract service interface: Based on the research of GIS web services, frequently used GIS web services have been classified as abstract service interfaces, the main classification of: tiled map service, name search service, network analysis service, data search service, topological analysis service etc. Abstract service interfaces are very important, because they separated platform application service interfaces from underlying GIS software, and the platform services don't depend on the specific GIS software. As required, the user could replace any of the basic GIS software during the construction process of public platform and digital city. However, Secondary development users of platform are using the platform service interface and would not find these changes. So they do not need to change their business GIS systems.

Service registration center: This paper designed unified registration center of SG and MS. User (administrator) can register all types of SG and MS. This descriptions are expressed by the information in the form of a unified XML, then description database of MS will been built. Finally, system will add MS to different SG by reading the description of these MS. Therefore, the service registration center lays the foundation for service aggregation.

GIS service group: A service group consisted of same type of GIS web services, for example service group of tiled map service, service group of name search service, service group of network analysis service, service group of data search service, service group of topological analysis service etc. A service group has one or more of the same GIS member services.

Service aggregation process model: According to the user's requirements of the aggregated service and type of $\mathrm{SG}$, it can aggregate a variety of GIS web services to meet the needs of users, such as single aggregation service (tiled map service, name search service, network analysis service, data search service etc.), composite aggregation service (searching place names within 500 meters around a road). Single aggregation service is mainly used for the basic aggregation of GIS web services among national, provincial and municipal. As name search service, principles of giving priority to the level of detail, it searches 1:50000 name database at the national level, and searches 1:10000 name database in provincial services, searches 1:2000 and 1:500 name databases. Finally, it comprehensive trades-off and returns the final result to the user. Composite aggregation service is mainly used for aggregation of GIS web 
services among the horizontal and the various government departments. For example, Provincial Bureau of Statistics provides detailed statistics for boroughs. By service aggregation process model, services aggregate the basic GIS web service to statistics thematic data service, and provide statistical services of thematic map.

In short, by the design and programming of specific service aggregation process model, specific software modules and aggregation library are realized, and add them to software architecture of aggregation service. Finally, various aggregation service instances are realized.

\section{QUALITY EVALUATION MODEL OF AGGREGATION SERVICE DESIGN}

\subsection{QoS of GIS member service}

Quality of Service (QoS) is to describe a product or service's ability to meet consumer demand. QoS is the key of which member service of GIS can be successfully used by service aggregation instances or not. It became a hot research that how to ensure the best member service to be provide for service instances.

QoS of GIS web service is defined from three aspects, i.e. GIS Web Service itself, networking conditions and service consumer. So it can be expressed as a six-tuple. QoS $=$ (Time, Cost, Reliability, Bandwidth, Server Capability, Throughput Capacity).

$>$ Execution Time (T): represents spent time from calling the service request to getting the results. Execution time includes time of processing user's service request $(\mathrm{Tp})$ and transmission time (Ts) on the network. That is $T=T p+T s$. Execution time is the most important parameters of the dynamics quality of GIS web services. It has a dynamic and uncertain characteristics, and is affected from many aspects, such as server performance, network transportation etc.

$>$ Execution cost (C): represents request cost of consumers calling GIS web services. The price of services is given by the service provider when the services registration. Therefore, this parameter is a static parameter of GIS web services and is assigned a certain percentage of all weights in the evaluation model.

> Reliability (R): represents the success probability of calling and executing GIS web service. Reliability associates with GIS web service host hardware, software and network connection status set etc. Reliability is the most important parameters of the dynamics quality of GIS web services.

> Bandwidth (B): represents the current available bandwidth of GIS web service host network. Bandwidths can real-time access according to monitoring information of resource information service. This parameter is a dynamic parameter of GIS web services.

$>$ Server Capability (SC): represents Performance information about service host, and includes Processor occupation (PO) of CPU and Remainder memory (RM). This parameter is a dynamic parameter of GIS web services.
$>$ Throughput Capacity (TC): represents the maximum number users of service. Throughput Capacity (TC) is given by the service provider when the services registration. This parameter is a static parameter of GIS web services.

\subsection{QoS-based selection of the best GIS services:}

Before service aggregation process automatically selected the best member service, it firstly filtrated the member services according to the QoS constraints which user has been given in advance. Then it used the method of Multiple Attribute Decision Making (MADM) to choose the best member services. The whole solution process is divided into three steps:

1) filtering the QoS according to the user's requirements For example, after filtering the QoS based on the user's requirements, there have $\mathrm{n}$ services to meet User's QoS constraints. That $\mathrm{S}=\left\{\mathrm{s}_{1}, \mathrm{~s}_{2}, \ldots, \mathrm{s}_{\mathrm{n}}\right\}$. QoS parameters of service can form a 6-dimensional tuples. QoS parameters of $\mathrm{N}$ services can be a multi-attribute (multi-dimensional QoS) decision making matrix Q. The rows of Q correspond to the QoS parameters of each service, the columns of Q represent a dimension of service QoS model (equation 1).)。

2) Quality factors of QoS Normalization process Different quality factors may have different quality characteristics, such as different units of measure, different types of range and antagonist of quality factors evaluation (The bigger values of factor are the better, contrary, the smaller values of factor are the better.). So different quality factors of services must be normalized. 。 Each quality factor of OoS could be divided into negative and positive factors. Negative factors refers to service QoS parameters which the higher value the worse quality. For example Execution time, Execution cost etc. Positive factors refers to service QoS parameters which the higher value the better quality. Such as Reliability, Bandwidth, Server Capability, Throughput Capacity etc. Negative factors could be normalized by the equation 2; Positive factors could be normalized by the equation 3 .

Where is the maximum value of the $\mathrm{j}$-dimensional in $\mathrm{Q}$, that. is the minimum value of the $\mathrm{j}$-dimensional in $\mathrm{Q}$, that . $\mathrm{Q}^{*}$ has been required after $\mathrm{Q}$ is normalized (the equation 4).

3) Score calculation

Based on normalization and information of user's preference, quality of each service could be calculated. User's preference describes level of interested in each QoS factor of the service. For example, the user preference information: $\mathrm{W}=\{\mathrm{w} 1, \ldots, \mathrm{w} 6\}$, that . Quality score value of each service could be calculation by the equation 5 .

\subsection{Quality Evaluation Model of Aggregation Service Implementation Mechanism}

In the service aggregation process model, in order to improve the robustness and effectiveness of aggregated services, Quality evaluation model of aggregation service can be divided into these processes: before running, in running, after running (shown in Fig. 2.).

$>$ Before the aggregation service running, aggregation service selection engine first chooses the most effective member service to aggregate in the same member services of SG.

$>$ In the aggregation service running, aggregation service execution engine calls the most effective member service in SG to complete service 
aggregation in accordance with service aggregation process model.

$>$ After the aggregation service running, system adds the latest QoS information records of member services to QoS database. In the next service aggregation instance execution, system can select the best member service in SG to aggregate. Therefore, aggregated services always use the best member service. The quality of service aggregation is ensured.

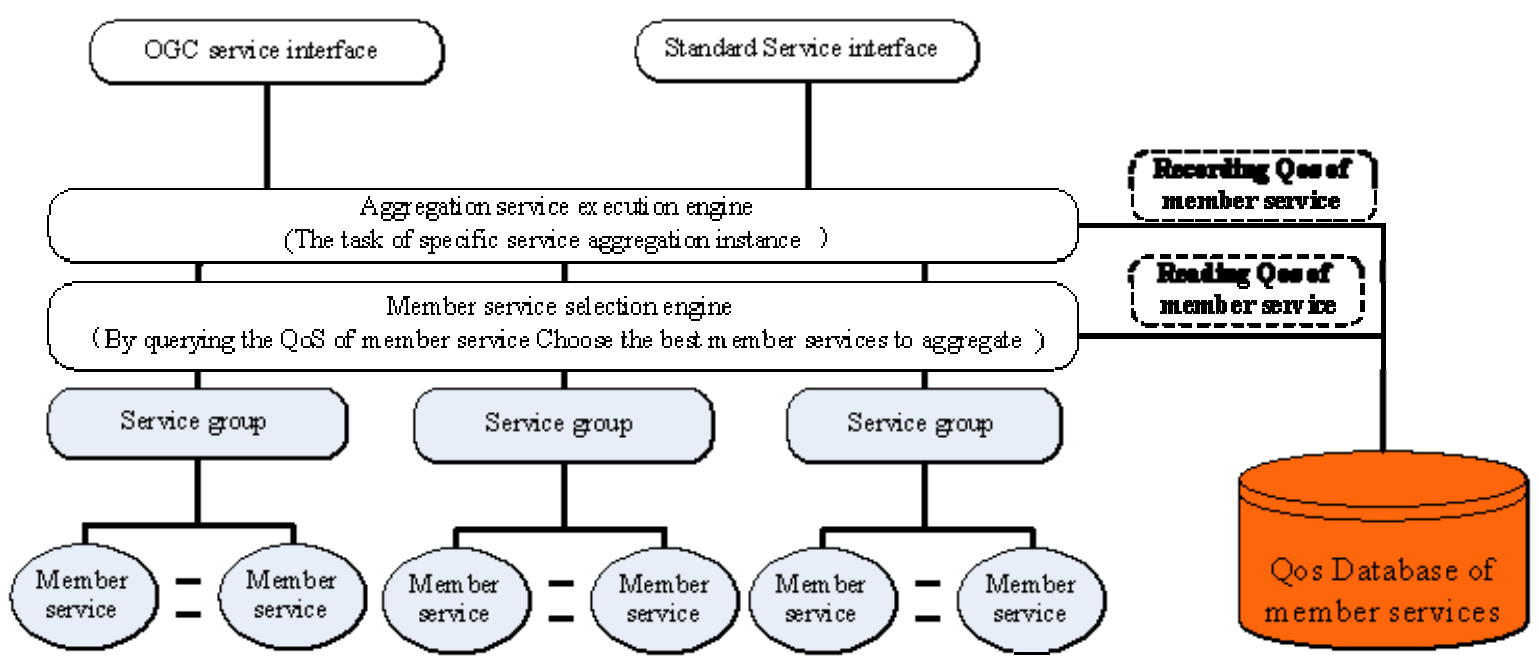

Fig.2. Logic diagram of Quality evaluation model of aggregation service implementation mechanism

\section{SERVICE AGGREGATION APPLICATION EXAMPLES}

Using .Net framework, this software architecture of GIS web service aggregation service has been realized and it has been widely used in public platform of GIS web service and a number of geo-spatial framework constructions for digital city in Sichuan Province. Social and economical benefits have been achieved and effectiveness of this software architecture is validated.

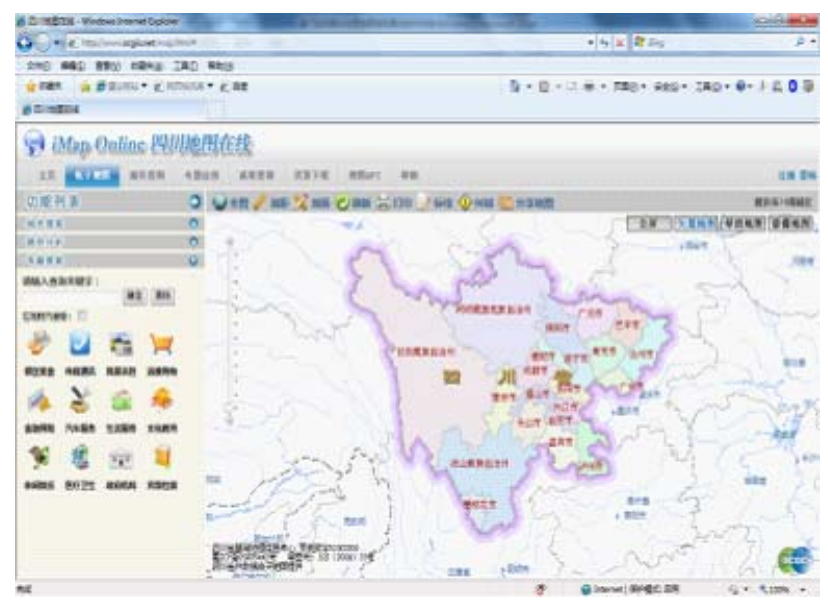

Fig.3. GIS web service of provincial platform aggregating World Map (national platform)

As a provincial public service node of National Public Platform of Geo-spatial Service construction, public platform of GIS web service in Sichuan, first, needs to aggregate GIS web services of National Public Platform of Geo-spatial Service (world map). Second, needs to aggregate GIS web services of municipal public platform of geo-spatial service (Information Base). Aggregation example is shown in Fig. 3.

\section{CONCLUSION}

According to requirements of National Public Platform of Geospatial Service and problems among the aggregation of heterogeneous GIS services, the paper designed software architecture of GIS web service aggregation based on SG. Using Abstract service interface, the software architecture converted a variety of heterogeneous services to a unified interface of GIS services, and separated Service aggregation model from the underlying GIS software. Users could not understand what the underlying member services have been called, only a URL would be able to call GIS web service aggregation. On this basis, quality evaluation model of Aggregation service was designed. Therefore, robustness and effectiveness of aggregated GIS web service was improved. The software architecture has been widely used in public platform of GIS web service and a number of geo-spatial framework construction for digital city in Sichuan Province, Applications of items showed that this software architecture was practicability.

\section{REFERENCES}

[1] Wang Y, Dai GP, HOU YR. Dynamic Methods of Trustaware Composite Service Selection. Journal of Computers, 2009, 32(8):1668-1675(in Chinese)

[2] Liu SL, Liu YX, Zhang F, Tang GF, Jing N. A dynamic Web services selection algorithm with QoS global optimal in Web services aggregation. Journal of Software,2007,18(3):646656(in Chinese) 
[3] Song FX, Liu JZ. Optimization of GIS Web Service Chaining Based On Qos. Journal of University of Electronic Science and Technology of China, 2010, 39(2):298-301(in Chinese)

[4] ISO9000.International Organization for Standardization. 2002,http://www.iso.ch/iso/en/iso9000/iso9000index.html

[5] Liangzhao Zeng, Boualem Benatallah, Marlon Dumas.Quality Driven Web Service Aggregation.In proceeding of WWW, Budapest, Hungary, pp411 421, 2003

[6] M.Kksalan and S.Zionts, editors. Multiple Criteria Decision Making in the New Millennium.Spinger-Verlag, 2001
[7] H.C.-L and K.Yoon. Multiple Criteria Decision Making. Lecture Notes in Economics and Mathematical Systems, Springer-Verlag, 1981

\section{CORRESPONDING AUTHOR}

LIU Jian-chuan, Sichuan Geomatics Center, Chengdu 610041, China

Tel.:13550318830, fax: +8602866065492, email:11411994@qq.com

Address: No7. JiuXinDaDao Road, GaoXin District, Chengdu, Sichuan, China 